\title{
Memory Impairment Induced by Borna Disease Virus 1 Infection is Associated with Reduced H3K9 Acetylation
}

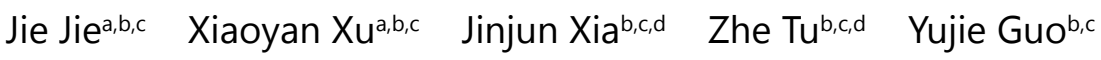 \\ Chenmeng Lib,c Xiong Zhang ${ }^{b, c}$ Haiyang Wang ${ }^{b, c}$ Weihong Song ${ }^{\mathrm{e}}$ Peng Xie $\mathrm{K}^{\mathrm{a}, \mathrm{b}, \mathrm{c}, \mathrm{d}}$ \\ aDepartment of Neurology, the First Affiliated Hospital, Chongqing Medical University, Chongqing, \\ ${ }^{b}$ Chongqing Key Laboratory of Neurobiology, Chongqing Medical University, Chongqing, 'Institute \\ of Neuroscience and the Collaborative Innovation Center for Brain Science, Chongqing Medical \\ University, Chongqing, 'Department of Neurology, Yongchuan Hospital, Chongqing Medical University, \\ Chongqing, China, eTownsend Family Laboratories, Department of Psychiatry, The University of British \\ Columbia, Vancouver, Canada
}

\section{Key Words}

Borna Disease Virus $1 \cdot$ Memory impairment $•$ H3K9 acetylation $•$ Synaptic plasticity

\begin{abstract}
Background/Aims: Borna disease virus 1 (BoDV-1) infection induces cognitive impairment in rodents. Emerging evidence has demonstrated that Chromatin remodeling through histone acetylation can regulate cognitive function. In the present study, we investigated the epigenetic regulation of chromatin that underlies BoDV-1-induced cognitive changes in the hippocampus. Methods: Immunofluorescence assay was applied to detect BoDV-1 infection in hippocampal neurons and Sprague-Dawley rats models. The histone acetylation levels both in vivo and vitro were assessed by western blots. The acetylation-regulated genes were identified by ChIP-seq and verified by RT-qPCR. Cognitive functions were evaluated with Morris Water Maze test. In addition, Golgi staining, and electrophysiology were used to study changes in synaptic structure and function. Results: BoDV-1 infection of hippocampal neurons significantly decreased H3K9 histone acetylation level and inhibited transcription of several synaptic genes, including postsynaptic density 95 (PSD95) and brain-derived neurotrophic factor (BDNF). Furthermore, BoDV-1 infection of Sprague Dawley rats disrupted synaptic plasticity and caused spatial memory impairment. These rats also exhibited dysregulated hippocampal H3K9 acetylation and decreased PSD95 and BDNF protein expression. Treatment with the HDAC inhibitor, suberanilohydroxamic acid (SAHA), attenuated the negative effects of BoDV-1. Conclusion: Our results demonstrate that regulation of H3K9 histone acetylation may play an important role in BoDV-1-induced memory impairment, whereas SAHA may confer protection against BoDV-1-induced cognitive impairments. This study finds important

J. Jie and X. Xu contributed equally to this work.

Peng Xie

and Weihong Song

Department of Neurology, the First Affiliated Hospital, Chongqing Medical University,

Chongqing (China)

E-Mail xiepeng@cqmu.edu.cn; weihong@mail.ubc.ca
\end{abstract}




\section{Cellular Physiology Cell Physiol Biochem 2018;49:381-394 and Biochemistry \begin{tabular}{l|l} 
DOI: 10.1159/000492890 & (c) 2018 The Author(s). Published by S. Karger AG, Basel \\
www.karger.com/cpb
\end{tabular} \\ Jie et al.: H3K9 Acetylation in Borna Disease Virus 1-induced Cognitive Impairment}

mechanism of BoDV-1 infection disturbing neuronal synaptic plasticity and inducing cognitive dysfunction from the perspective of histone modification.

(C) 2018 The Author(s)

Published by S. Karger AG, Basel

\section{Introduction}

Bornaviruses (Order Mononegavirales Family Bornaviridae) are phylogenetically diverse, nonsegmented, negative single-stranded RNA viruses [1-3]. Borna disease virus 1 (BoDV-1), as part of the species Mammalian 1 bornavirus, is a highly neurotropic prototype of Bornaviruses and infects a wide variety of mammal species [4, 5]. BoDV-1 was recently identified as the cause of human death after organ transplantation - the first hard evidence that BoDV-1 can actually infect humans and cause disease [6]. BoDV-1-infection may induce a large spectrum of neurological disorders ranging from immune-mediated acute encephalitis to non-inflammatory behavioral alterations [7, 8]. Previous studies demonstrated that BoDV-1 establishes a persistent infection in the hippocampus and limbic lobe of rodents in the neonatal period, which induced cognitive deficits and mental disorders $[4,9]$. Notwithstanding abundant discussion of BoDV-1-driven pathophysiology, the precise mechanism underlying how BoDV-1 disrupts memory is poorly understood.

Chromatin remodeling, especially via histone acetylation, plays a key role in hippocampusdependent cognitive function [10-12]. Memory formation and synaptic plasticity have been shown to be associated with changes in histone acetylation [13]. Aberrant activity of histone acetyl transferases (HATs) or histone deacetylases (HDACs) can alter the levels of acetylated histones and cause abnormal gene expression $[14,15]$. Suberanilohydroxamic acid (SAHA) is an HDAC inhibitor that targets Class I and Class IIb HDACs, causing increased acetylation [16]. Treatment with SAHA can enhance memory formation in rats and mice [17], and improves memory deficits in animal models of neurodegenerative disorders $[18,19]$. Infection with a laboratory BoDV-1 strain affects site-specific histone acetylation in cortical [20] and hippocampal neurons [21]. Moreover, our previous study showed that infection with a human BoDV-1 strain epigenetically impacts the human oligodendroglial (OL) cell proteome through histone acetylation [22]. However, the epigenetic regulation of chromatin that underlies BoDV-1-induced cognitive changes in the hippocampus remains unknown.

In this study we tested the hypothesis that BoDV-1 infection disturbs synaptic plasticity and induces cognitive dysfunction via histone acetylation. Here, we show that infection with a human BoDV-1 strain caused a dramatic decline in the level of H3K9 acetylation, and consequently reduced postsynaptic density 95 (PSD95) and brain-derived neurotrophic factor (BDNF) expression and impaired synaptic plasticity in hippocampal neurons. These changes contributed to BoDV-1-induced cognitive deficits, which were attenuated by the HDAC inhibitor, SAHA.

\section{Materials and Methods}

\section{Primary neuronal cultures and viral infection}

All animal experiments were approved by the Research Ethics Committee of ChongQing Medical University. Primary hippocampal neurons were prepared from Sprague Dawley rat embryos at gestational day 17 by the $0.125 \%$ trypsin (Thermo Fisher Scientific, Waltham, USA) dissociation method, as described previously [23]. At the end of this procedure, neurons were directly plated in six-well culture plates. Before seeding, supports were coated with $500 \mu \mathrm{g} / \mathrm{ml}$ poly-L-lysine (Sigma, St. Louis, USA). Neurons were maintained in Neurobasal medium supplemented with $100 \mu \mathrm{g} / \mathrm{ml}$ penicillin-streptomycin (Sigma), 0.5 mM glutamine, and 2\% B-27 supplement (Thermo Fisher Scientific). Half of each culture was infected with human BoDV-1 strain Hu-H1 [24] (kindly provided by Professor Hanns Ludwig, Free University of Berlin, Germany) $5 \mathrm{~h}$ after plating. Cultured primary neurons were supplemented with $1 \mu \mathrm{M}$ SAHA [25] (Sigma) for $48 \mathrm{~h}$ or $200 \mu \mathrm{M}$ favipiravir (T-705) [26] (Selleckchem, Houston, USA) for 7 days for follow-up experiments. 


\section{Cellular Physiology Cell Physiol Biochem 2018;49:381-394 \begin{tabular}{ll|l} 
and Biochemistry Published onlIne: 23 August, 2018 & $\begin{array}{l}\text { (c) } 2018 \text { The Author(s). Published by S. Karger AG, Basel } \\
\text { www.karger.com/cpb }\end{array}$ \\
\hline
\end{tabular} \\ Jie et al.: H3K9 Acetylation in Borna Disease Virus 1-induced Cognitive Impairment}

\section{Immunofluorescence assay}

Cells were fixed for $30 \mathrm{~min}$ at room temperature with $4 \%$ paraformaldehyde followed by permeabilization for $5 \mathrm{~min}$ in $0.25 \%$ Triton X-100. After permeabilization, cells were rinsed with phosphatebuffered saline (PBS, Hyclone, Logan, USA) and blocked with 5\% bovine serum albumin for 30 min. Cells were then incubated for $12 \mathrm{~h}$ with anti-BoDV-1-specific p24 and p40 protein primary monoclonal antibody (1:200; GenScript, Nanjing, China) or anti-MAP2 antibody (1:2000; Abcam, Cambridge, UK) at $4^{\circ} \mathrm{C}$, followed by incubation with secondary antibodies for $2 \mathrm{~h}$ at room temperature. After several washes with PBS, the cells were counterstained with DAPI. Immunofluorescence was detected using an inverted fluorescence microscope (Nikon, Tokyo, Japan).

\section{Protein extraction and western blot analysis}

Proteins were extracted and western blots performed. For each sample, $30 \mu \mathrm{g}$ total protein was separated on a $10 \%$ SDS-polyacrylamide gel and transferred electrophoretically to a PVDF membrane. Membranes were blocked with 5\% nonfat dried milk in TBST and were incubated overnight with the following antibodies: anti-GAPDH (Abcam) at 1:10, 000, anti-acetyl histone H2B (Lys-5, K5, CST, Boston, USA) at 1:1000, anti-acetyl histone H2B (Lys-20, K20, CST) at 1:1000, anti-acetyl histone H3 (Lys-9, CST) at 1:1000, anti-acetyl histone H3 (Lys-14, CST) at 1:500, anti-acetyl histone H4 (Lys-16, K16, CST) at 1:1000, anti-PSD95 (CST) at 1:1000, anti-BDNF (Beyotime, China) at 1:500, anti-PUM2 (CST) at 1:800, anti-VAMP-2 (CST) at 1:1000, anti-SYN1 (CST) at 1:1000, and anti-DRD1 (Sigma) at 1:2000. Horse radish peroxidase-conjugated secondary antibodies were used and detected using Western Lighting Western Blot Chemiluminescence (NEL 104001EA; Perkin Elmer).

\section{ChIP-seq analysis}

Chromatin immunoprecipitation (ChIP) experiments were performed as described previously [27]. Briefly, two hippocampal samples for each group were individually cross-linked with $1 \%$ formaldehyde, quenched with $0.125 \mathrm{M}$ glycine, and spun down at $1000 \mathrm{~g}$ for $5 \mathrm{~min}$ at $4^{\circ} \mathrm{C}$. To isolate chromatin, samples were washed and homogenized with a Dounce homogenizer in $0.5 \mathrm{ml}$ cell lysis buffer containing proteinase and phosphatase inhibitors. Samples were centrifuged at $1000 \mathrm{~g}$ for $5 \mathrm{~min}$ and homogenized again in $0.5 \mathrm{ml}$ nuclear lysis buffer with inhibitors. DNA was sheared using a sonication instrument (Scientz, Ningbo, China) to a fragment length of 200-500 bp. Total genomic DNA (input) was quantified and $20 \mu \mathrm{g}$ of chromatin from each sample was immunoprecipitated overnight at $4^{\circ} \mathrm{C}$ with $10 \mu \mathrm{l}$ anti-acetyl-H3K9 (CST), $1 \mu \mathrm{l} \mathrm{normal}$ mouse IgG (12-371B; Millipore, MA, USA) as a negative control, or $1 \mu \mathrm{l}$ anti-RNA polymerase II (05-623B; Millipore) as a positive control. After incubation, nucleosome complexes were isolated with $30 \mu \mathrm{l}$ Magnetic Protein A/G Beads (CS204457; Millipore) for $2 \mathrm{~h}$ at $4^{\circ} \mathrm{C}$. The complexes were washed and dissociated from the beads by incubation in nuclear lysis buffer containing $1 \% \mathrm{SDS}$ at $65^{\circ} \mathrm{C}$ for $2 \mathrm{~h}$. Histones were then digested with proteinase $\mathrm{K}$ for $2 \mathrm{~h}$ at $65^{\circ} \mathrm{C}$, after which the DNA was finally extracted and purified using spin columns. DNA concentrations were measured on a Nanodrop spectrophotometer (Thermo Fisher Scientific). The uniformity of fragment size was validated and quality control was performed on a 2100 BioAnalyzer (Agilent Technologies, CA, USA).

Different combinations of library preparation kits and barcodes were used for library preparation, and libraries were prepared in accordance with the manufacturer's instructions. ChIP-seq libraries were prepared from 5-15 ng ChIP DNA or $100 \mathrm{ng}$ input DNA and sequenced using the Illumina HiSeq 2500 system.

\section{Cell RNA extraction and real-time polymerase chain reaction (RT-qPCR)}

RNA from hippocampal neurons was extracted with TRIzol, after which reverse transcription with a PrimeScriptTM RT Reagent Kit (Takara, Tokyo, Japan) was performed. RT-qPCR was carried out using SYBR-Green-based reagents (Invitrogen, California, USA) with a LightCycler® 96 Real-Time qPCR Detection system (Roche, Basel, Switzerland). Thermocycling conditions consisted of an initial denaturation step for $10 \mathrm{~min}$ at $95^{\circ} \mathrm{C}$, followed by 40 cycles of $95^{\circ} \mathrm{C}$ for $30 \mathrm{~s}$ and $58^{\circ} \mathrm{C}$ for $45 \mathrm{~s}$. The relative quantities of amplified product were calculated using the comparative cycle threshold (Ct) method. Data were derived from three independent amplifications. The primer sequences used for real-time PCR are listed in Table 1. GAPDH was used as a reference gene. 


\section{Cellular Physiology Cell Physiol Biochem 2018;49:381-394 \begin{tabular}{l|l|l} 
and Biochemistry & DOI: 10.1159/000492890 & $\begin{array}{l}\text { C } 2018 \text { The Author(s). Published by S. Karger AG, Basel } \\
\text { www.karger.com/cpb }\end{array}$
\end{tabular} \\ Jie et al.: H3K9 Acetylation in Borna Disease Virus 1-induced Cognitive Impairment}

\section{Animal model and treatments}

All behavioral experiments were performed under light conditions between 9:00 $\mathrm{h}$ and 16:30 h. All efforts were made to minimize the number of animals used and their suffering. Within $12 \mathrm{~h}$ after birth, $30 \mu \mathrm{l}\left(10^{4} \mathrm{FFU} / \mathrm{ml}\right)$ of BoDV-1 (Hu-H1) or PBS was inoculated into the right cerebral hemisphere of Sprague Dawley rat pups. Seven days later, half of the BoDV-1-infected rats were intraperitoneally injected with SAHA (25 mg/kg) for a week. After weaning, control $(n=14)$, SAHA $(n=14)$, and mockinfected $(n=14)$ rats were separated and housed individually. The ratio of males and females was identical among the three groups. Behavioral testing was performed from postnatal day $42(\mathrm{P} 42)$ to $\mathrm{P} 49$ and then rats were euthanized at P50 for dissection and subsequent analysis.

\section{Behavioral testing}

The Morris water maze (MWM) test was conducted in a circular tank (diameter $1.8 \mathrm{~m}$ ) filled with opaque water. A platform $(11 \times 11 \mathrm{~cm})$ was submerged below the water surface in the center of the target quadrant. The whole test consists of a visible platform test (day 1), a hidden platform test (days 2-5) and a probe test 24 hours after the last hidden platform test (day 6). The swimming path of each rat was recorded using a video camera and analyzed using Smart software (v2.5.21). For each training session, the rats were placed into the maze at four random points in the tank. The rats were allowed to search for the platform for $90 \mathrm{~s}$. If they did not find it within this period, they were gently guided towards it and then allowed to remain on it for $15 \mathrm{~s}$. The latency to find the platform for each trial was recorded. During the memory test (probe test), the platform was removed from the tank and the rats were allowed to swim in the maze for $60 \mathrm{~s}$.

\section{Golgi staining and analysis}

For Golgi staining, we used the FD Rapid Golgi Stain Kit (FD NeuroTechnologies, Ellicott City, MD, USA). Dissected rat brains were immersed in Solutions A and B for 2 weeks at room temperature and then transferred into Solution C for 72h. Brains were sectioned at $100 \mu \mathrm{m}$ thickness using a VT1000S Vibratome (Leica). These sections were then placed in a mixture of 1 part Solution D, 1 part Solution E, and 2 parts double-distilled water for $10 \mathrm{~min}$, after which the sections were dehydrated and cleared in xylene. Images of dendrites were then acquired using an optical microscope (Nikon). Dendrite branches were reconstructed using Image J software and subjected to Sholl analysis. Spine density was also measured using Image J. All morphological analyses were performed by individuals blinded to the genotypes and experimental conditions.

\section{Electrophysiology}

Rats were deeply anesthetized with urethane $(1.5 \mathrm{~g} / \mathrm{kg}$, i.p.) and transcardially perfused with N-methylD-glucamine (NMDG) artificial cerebral spinal fluid (ACSF) prior to decapitation as described previously [28]. The hippocampus was rapidly dissected and acute coronal hippocampal slices $400 \mu \mathrm{m}$ thick were cut using a vibratome (VT1000 S, Leica Microsystems) in ice-cold cutting solution (NMDG ACSF) bubbled with $5 \% \mathrm{CO}_{2}$ and $95 \% \mathrm{O}_{2}$. The slices were then incubated in oxygenated ACSF for $2 \mathrm{~h}$ at $30^{\circ} \mathrm{C}$. Next, the slices were transferred into a recording chamber filled with normal ACSF. Field excitatory postsynaptic potentials (fEPSPs) evoked by stimulation of the Schaffer collateral/commissural pathway were recorded in the hippocampus with pipettes filled with ACSF (1-2 M $\Omega$ ). Test fEPSPs were evoked at a frequency of $0.033 \mathrm{~Hz}$ and at a stimulus intensity that was adjusted to approximately $50 \%$ of the intensity that elicited the maximal response. After a stable $30 \mathrm{~min}$ baseline recording of fEPSPs, long-term potentiation (LTP) was induced 


\section{Cellular Physiology Cell Physiol Biochem 2018:49:381-394 and Biochemistry \begin{tabular}{l|l} 
DOI: 10.1159/000492890 & (c) 2018 The Author(s). Published by S. Karger AG, Basel \\
www.karger.com/cpb
\end{tabular} \\ Jie et al.: H3K9 Acetylation in Borna Disease Virus 1-induced Cognitive Impairment}

by high-frequency stimulation $(100 \mathrm{~Hz}$ for $1 \mathrm{~s})$. After a $30 \mathrm{~min}$ stable baseline, all data acquisition (filtered at $3 \mathrm{kHz}$ and digitized at $10 \mathrm{kHz}$ ) was performed using PatchMaster v2.73 software (HEKA Electronic, Lambrecht/Pfalz, Germany).

\section{Statistical analysis}

All data were analyzed using SPSS version 21.0 for Windows (SPSS Inc., USA). Data are expressed as the mean \pm SEM. Comparisons among experimental groups were performed using the unpaired t-test and behavioral data were analyzed using one-way ANOVA, followed by Bonferroni's test and Dunnett's T3 test when necessary. In all comparisons, P-values less than 0.05 were considered to indicate statistical significance.

\section{Results}

\section{BoDV-1 infection decreases histone acetylation of $H 3 K 9$}

To assess the impact of BoDV-1 infection on histone acetylation, primary neuronal cultures were infected with BoDV-1 and then cultured for 9 days to allow all neurons to be infected with BoDV-1 (Fig. 1A). Acetylation of H2, H3, and H4 was assessed by western blots using antibodies targeting lysine residues that are known to be acetylated (Fig. 1B). BoDV1 infection led to a significant reduction of H3K9 acetylation (58.19\% of control, P<0.01). There was no difference in the acetylation of H2BK5, H2BK20, H3K14, and H4K16 between the BoDV-1-infected neurons and controls (Fig. 1C).

\section{BoDV-1 infection inhibited the expression of $\mathrm{H} 3 \mathrm{~K} 9$ acetylation-mediated synaptic plasticity-} related genes

The hippocampus is an important target of BoDV-1 infection [29, 30]. Neuronal plasticity plays an important role in cognition. To investigate whether deregulated H3K9 acetylation affects memory formation and synaptic plasticity, we applied ChIP-seq technology to analyze H3K9 acetylation within gene promoter regions that were defined as 1 $\mathrm{Kb}$ upstream and $1 \mathrm{~Kb}$ downstream of the transcription startsite (TSS). The resulting reads were mapped to a reference rat genome. Compared with the control group, 808 genes showed reduction of H3K9 acetylation after BoDV-1 infection. The genes with remarkable loss of H3K9ac are shown in Table $2(\log 2 \mathrm{FC}<-1$, $\mathrm{P}<0.05)$. Of these, we found a number of memory formation or synaptic plasticityassociated genes, including PSD95, BDNF, PUM2, VAMP2, SYN1 and DRD1. Chip-Seq analysis confirmed BoDV-1-mediated loss of $\mathrm{H} 3 \mathrm{~K} 9$ acetylation in the promoters of these genes (Fig. 2A). Acetylation of H3K9 indicates an open chromatin state, and the enrichment of H3K9ac near a TSS is closely related to the transcriptional activity of the gene [31]. In BoDV-1infected neurons, RT-qPCR and western

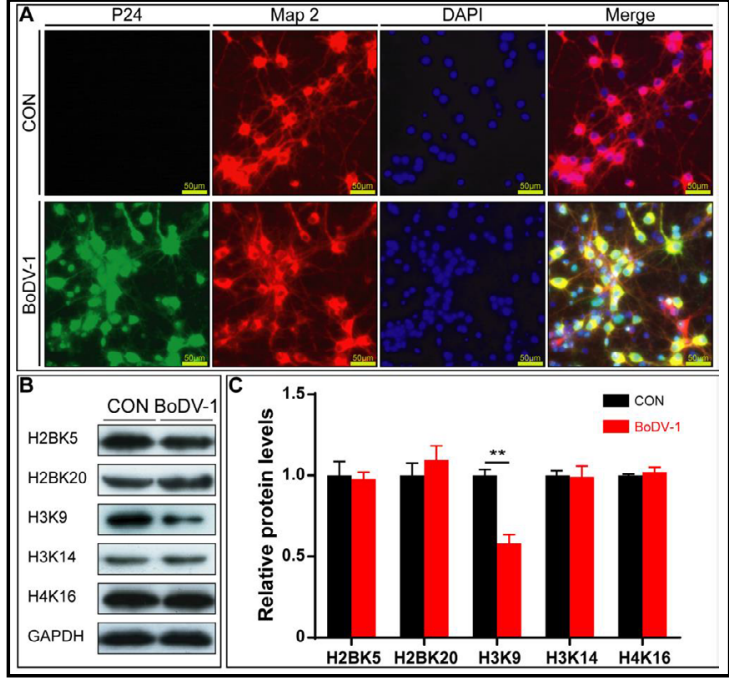

Fig. 1. BoDV-1 infection decreases histone acetylation levels of H3K9 in primary hippocampal neurons. (A) BoDV-1 infection was measured by IFA. BoDV-1 P24 detected with a primary monoclonal antibody (green), Neurons marked with chicken polyclonal MAP-2 (red), Nuclei stained with DAPI (blue), merged image (scale bars: $50 \mu \mathrm{m}$ ). (B) Levels of histone acetylation were evaluated by Western blots for H2, H3, and H4. (C) Quantification of histone acetylation levels. All data are presented as mean \pm SEM. Statistical analysis was performed by unpaired t-test. ${ }^{*} \mathrm{P}<0.05$, ${ }^{* *} \mathrm{P}<0.01$ vs. controls. 


\section{Cellular Physiology

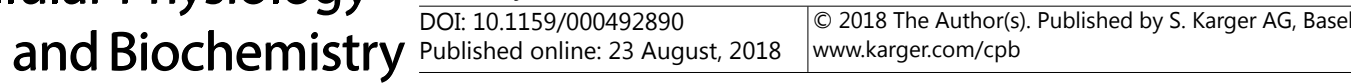

Jie et al.: H3K9 Acetylation in Borna Disease Virus 1-induced Cognitive Impairment

Table 2. CHIP-seq shows genes with H3K9 hypoacetylation on TSS

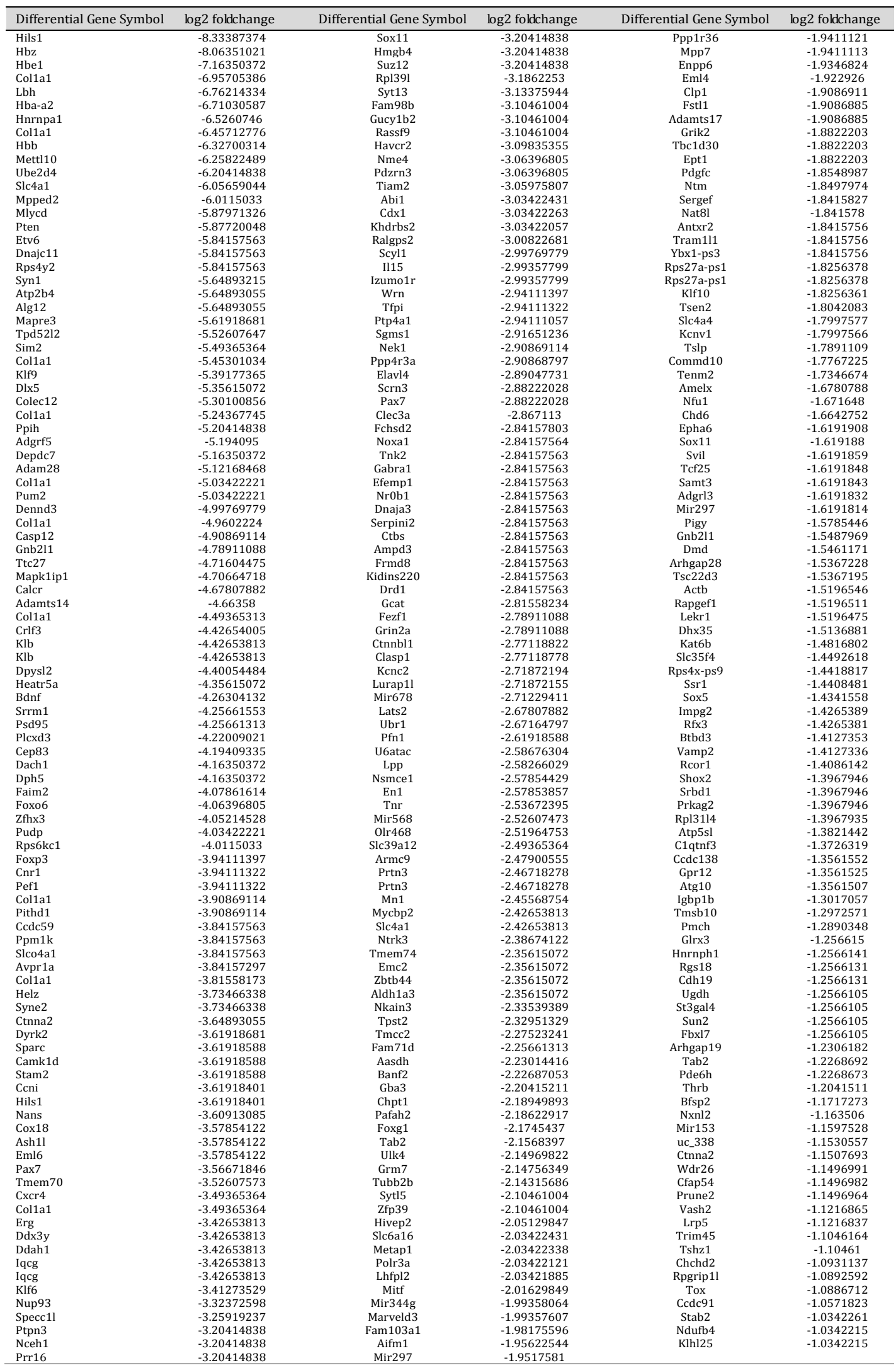


blot analysis confirmed that the expression of these genes was also reduced both at mRNA (PSD95: $48.62 \%$ of control, BDNF: $63.74 \%$ of control, $\mathrm{P}<0.01$ ) and protein levels (PSD95: $62.37 \%$ of control, BDNF: $57.25 \%$ of control, $\mathrm{P}<0.01$ ) (Fig. 2B-D). Treatment with the HDAC inhibitor, SAHA, nearly reversed the expression levels of PSD95 and BDNF back to normal. Although the expression levels of other genes were upregulated after the use of SAHA, they were significantly lower than their normal levels, indicating that their expression was affected by histone acetylation to some extent and that other

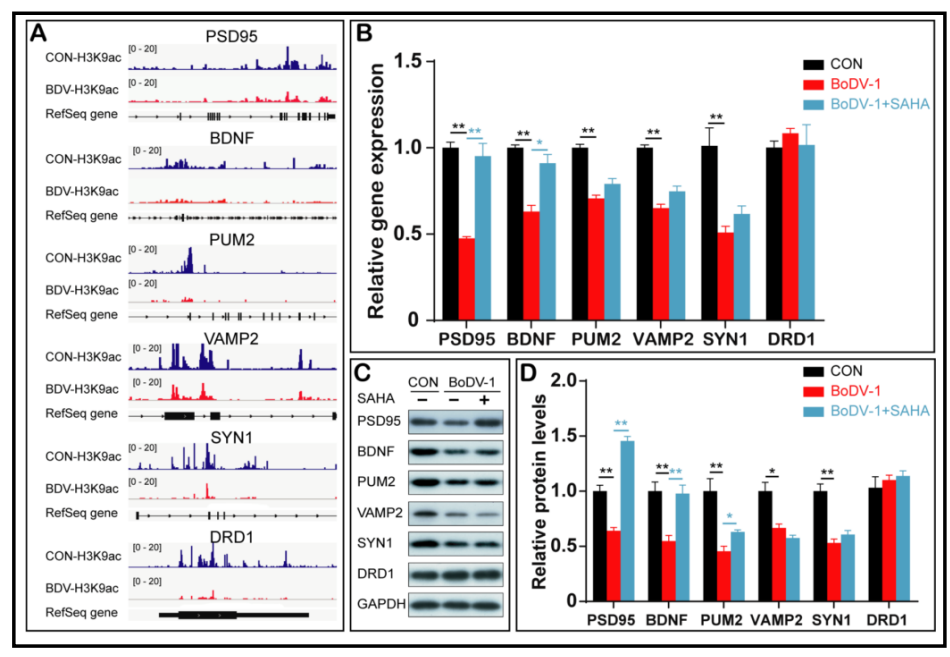

Fig. 2. BoDV-1 infection inhibited the expression of H3K9 acetylationmediated synaptic plasticity-related genes. (A) ChIP-Seq analysis of H3K9ac at PSD95, BDNF, PUM2, VAMP2, SYN1 and DRD1 promoters in primary hippocampal neurons. (B-D) RT-qPCR and Western blot analysis of differential expression of PSD95, BDNF, PUM2, VAMP2, SYN1 and DRD1 during BoDV-1 and SAHA treatment. All data are presented as mean \pm SEM. Statistical analysis was performed by unpaired $t$-test. ${ }^{*} \mathrm{P}<0.05,{ }^{* *} \mathrm{P}<0.01$ vs. controls. mechanisms were involved in their regulation. These findings demonstrated that BoDV-1-mediated hypoacetylation of H3K9 may be the main mechanism by which BoDV-1 suppressed the transcription of PSD95 and $B D N F$.

SAHA does not inhibit the proliferation of BoDV-1 and rescues the BoDV-1-induced reduction of $\mathrm{H} 3 \mathrm{~K} 9$ acetylation

To explore whether SAHA could affect the replication of BoDV-1, we used T-705, a virus-inhibiting drug, as a positive control and observed the effect of SAHA on BoDV-1 by immunofluorescence and RT-qPCR experiments (Fig. 3A-B). The results showed that the number of virus copies in neurons treated with T-705 was significantly reduced $(55.45 \%$ of BoDV-1 group, $\mathrm{P}<0.01$ ), whereas the virus numbers in the control and SAHA-treated neurons were normal. This shows that SAHA does not interfere with the replication of BoDV-1 and that T-705 can significantly inhibit viral replication. In addition, we also observed the effects of SAHA and T-705 on H3K9 acetylation levels in BoDV-1-infected neurons (Fig. 3C). Western blots showed that SAHA significantly increased H3K9 acetylation levels $(181.76 \%$ of BoDV-1 group, $\mathrm{P}<0.01$ ) compared to the $\mathrm{T}-705$-treated group $(154.88 \%$ of BoDV-1 group, $\mathrm{P}<0.01)$. These results suggest that SAHA counteracts the damaging effects of BoDV-1 on synaptic plasticity by altering the level of $\mathrm{H} 3 \mathrm{~K} 9$ acetylation, rather than by inhibiting viral replication.

\section{BoDV-1 infection impairs spatial learning in rats}

To determine the effects of BoDV-1 and SAHA on spatial learning and memory, rats were subjected to a Morris water maze test (Fig. 4A-B). In the visible platform test, the three groups had similar escape latency (CON: $70.57 \pm 4.94$, BoDV-1: $73.14 \pm 4.81$ and SAHA: 69.29 $\pm 4.14 \mathrm{~s}, \mathrm{P}>0.05$ ) (Fig. $4 \mathrm{C}$ ), indicating that there was no motor or vision dysfunction in BoDV1 or SAHA rats.

In the hidden platform test from day 2 to day 5 , the escape latency was significantly longer for the BoDV-1 rats compared with the control group (71.45 \pm 3.00 vs. $50.84 \pm 4.49 \mathrm{~s}$ on day $2,63.41 \pm 4.06$ vs. $31.63 \pm 3.52$ s on day 3 and $55.79 \pm 4.03$ vs. $26.61 \pm 2.42$ s on day 4 , $50.16 \pm 4.19$ vs. $22.34 \pm 2.70$ s on day $5, \mathrm{P}<0.01$ ), and SAHA treatment significantly shortened 


\section{Cellular Physiology and Biochemistry

Fig. 3. SAHA does not inhibit the proliferation of BoDV-1 and rescues BoDV-1-reduced H3K9 acetylation. (A) BoDV-1 infection was measured by IFA. BoDV-1 P40 was detected with a primary monoclonal antibody (red), nuclei were stained with DAPI (blue), merged image (scale bars: $50 \mu \mathrm{m}$ ). (B) RT-qPCR detection of P40 gene expression. (C) Western blot detection of acetylated H3K9. GAPDH was used as a loading control. All data are presented as the mean \pm SEM. Statistical significance was determined using the unpaired t-test. ${ }^{* *} \mathrm{P}<0.01$ vs. controls.

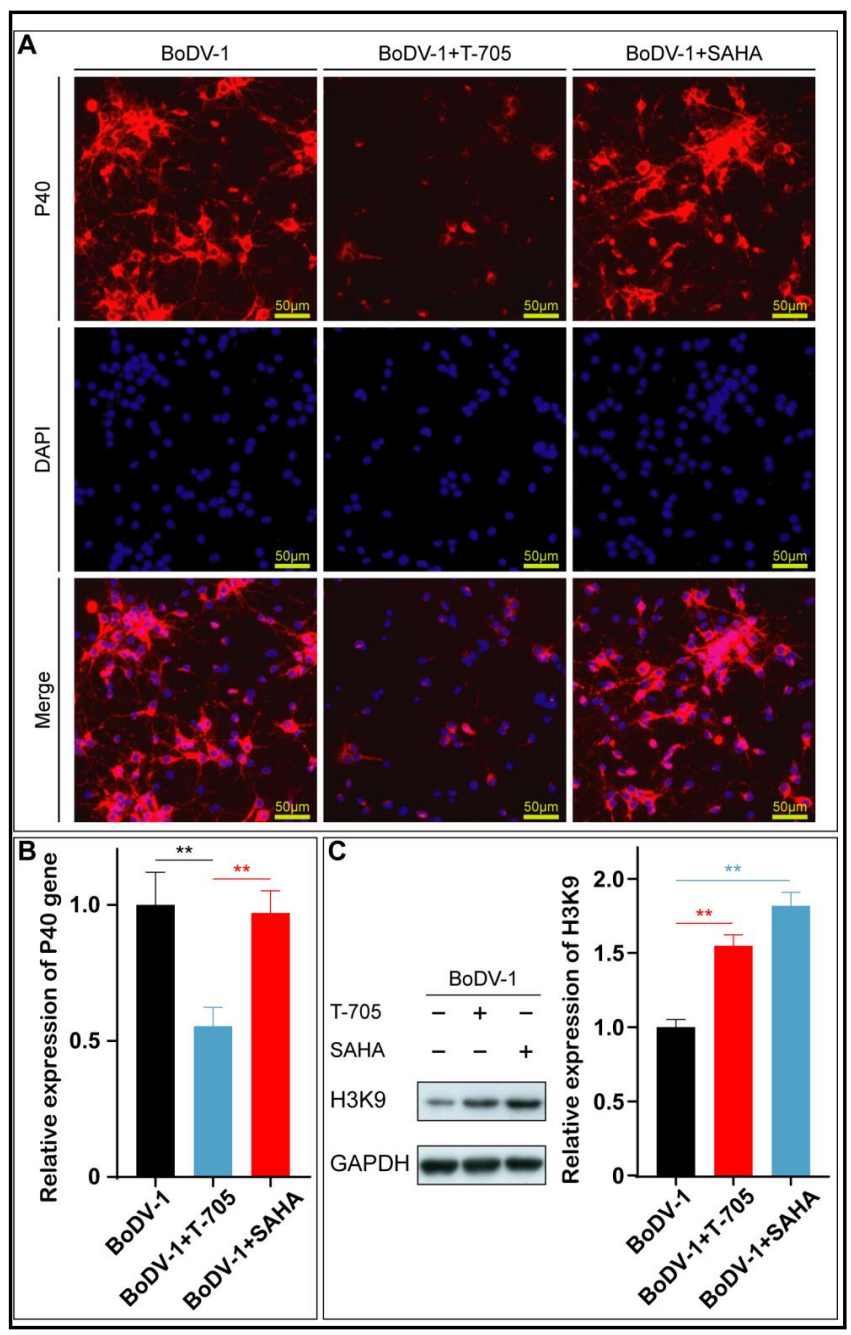

Fig. 4. BoDV-1 infection impairs spatial learning in rats and SAHA treatment recovers cognitive ability. (A) Animal experiment process design. (B-F) Morris water maze test. (C) In the visible platform tests, the latency of escape onto the platform. (D) In the hidden platform test, the escape latency on the $2^{\text {nd }}, 3^{\text {rd }}, 4^{\text {th }}$ and $5^{\text {th }}$ day. (E) The tracking of rats activity during the $90 \mathrm{~s}$ trial on the $5^{\text {th }}$ day. (F) In the probe trial, the crossing times of the target quadrant. All data are presented as mean \pm SEM (14 rats per group). Statistical analysis was performed by one-way ANOVA. ${ }^{*} \mathrm{P}<0.05,{ }^{* *} \mathrm{P}<0.01$ vs. controls.

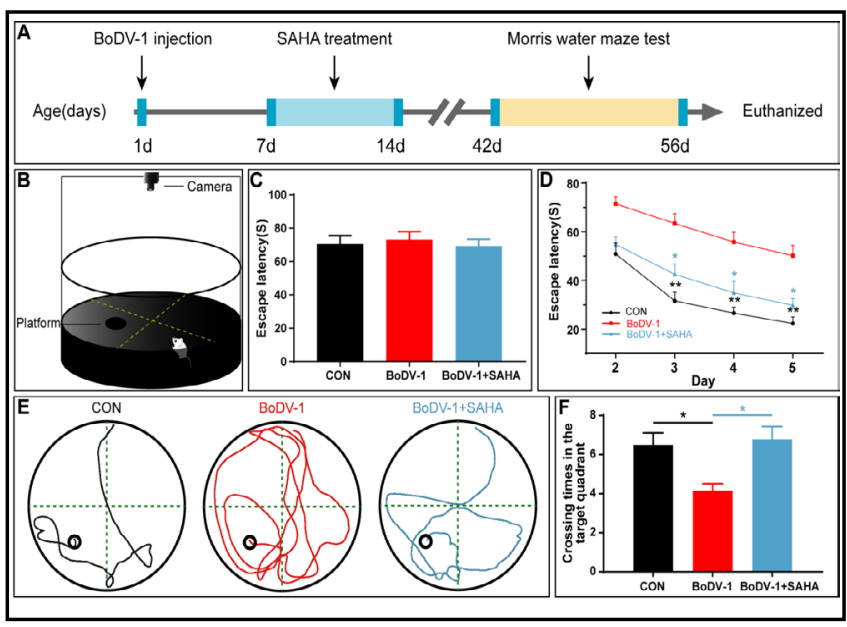

the escape latency induced by BoDV-1 (54.75 \pm 3.10 vs. $71.45 \pm 3.00$ s on day $2,42.51 \pm 4.33$ vs. $63.41 \pm 4.06$ s on day 3 and $34.89 \pm 4.78$ vs. $55.79 \pm 4.03$ s on day $4,29.79 \pm 2.76$ vs. $50.16 \pm$ 4.19 s on day $5, \mathrm{P}<0.05$ ) (Fig. 4D). Traces of the movement of rats during the hidden platform test are presented in Fig. 4E. In the probe trial on the last day of testing, BoDV-1 infection resulted in fewer crosses of the target quadrant ( $4.14 \pm 0.38 \mathrm{vs} .6 .50 \pm 0.62 \mathrm{~s}, \mathrm{P}<0.05)$. However, 


\section{Cellular Physiology \begin{tabular}{ll|l} 
and Biochemistry Published onlIne: 23 August, 2018 & $\begin{array}{l}\text { (c) } 2018 \text { The Author(s). Published by S. Karger AG, Basel } \\
\text { www.karger.com/cpb }\end{array}$ \\
\hline
\end{tabular}

SAHA treatment improved the performance $(6.79 \pm 0.65$ vs. 4.14 $\pm 0.38 \mathrm{~s}, \mathrm{P}<0.05$ ) (Fig. 4F).

These results demonstrated that BoDV-1 infection significantly impaired cognitive function but SAHA rescued this impaired cognitive ability.

BoDV-1 infection decreases histone acetylation of $\mathrm{H} 3 \mathrm{~K} 9$ and affects synaptic protein expression in rats

Inordertoconfirm theinfection of BoDV-1 in the rat hippocampus, immunofluorescence assay was used for detection (Fig. 5A). Then, to confirm the effect of BoDV-1 infection on acetylation in vivo, we extracted proteins of rat hippocampus for Western blot analysis. BoDV-1 infection led to a significant decrease of $\mathrm{H} 3 \mathrm{~K} 9 \mathrm{ac}$ $(44.61 \%$ of control, $\mathrm{P}<0.01)$ (Fig. 5B-C), consistent with our previous findings in vitro. In addition, SAHA treatment induced an increase of acetylation on $\mathrm{H} 3 \mathrm{~K}$. We next examined the expression of key proteins related to synaptic plasticity. BoDV-1 infection significantly decreased PSD95 (78.49\% of control, $\mathrm{P}<0.01$ ) and BDNF (67.23\% of control, $\mathrm{P}<0.01$ ) expression in rats. SAHA treatment effectively restored the expression in the BoDV-1-infected rats (Fig. $5 \mathrm{D}-\mathrm{E})$. These results suggest that BoDV-1-induced H3K9 acetylation defect led to synaptic plasticity damage in vivo.

\section{BoDV-1 infection caused} abnormalities of synaptic plasticity in rats

To examine the effect of BoDV-1 on synaptic plasticity, the number of dendritic branches and neuronal spine density were examined by using Golgi staining. As shown in Fig. 6, the mean number of dendritic branches and neuronal dendritic spine density were sharply decreased in the hippocampus of the BoDV-1 group rats compared with those in normal control rats $(\mathrm{P}<0.05, \mathrm{P}<0.01)$. SAHA treatment significantly increased the number of dendritic branches and the neuronal spine density compared with those in the BoDV-1 group $(\mathrm{P}<0.05, \mathrm{P}<0.01)$. These findings suggest that BoDV-1 infection caused the structural damage of synaptic plasticity and the effect can be reversed by HDAC inhibition.

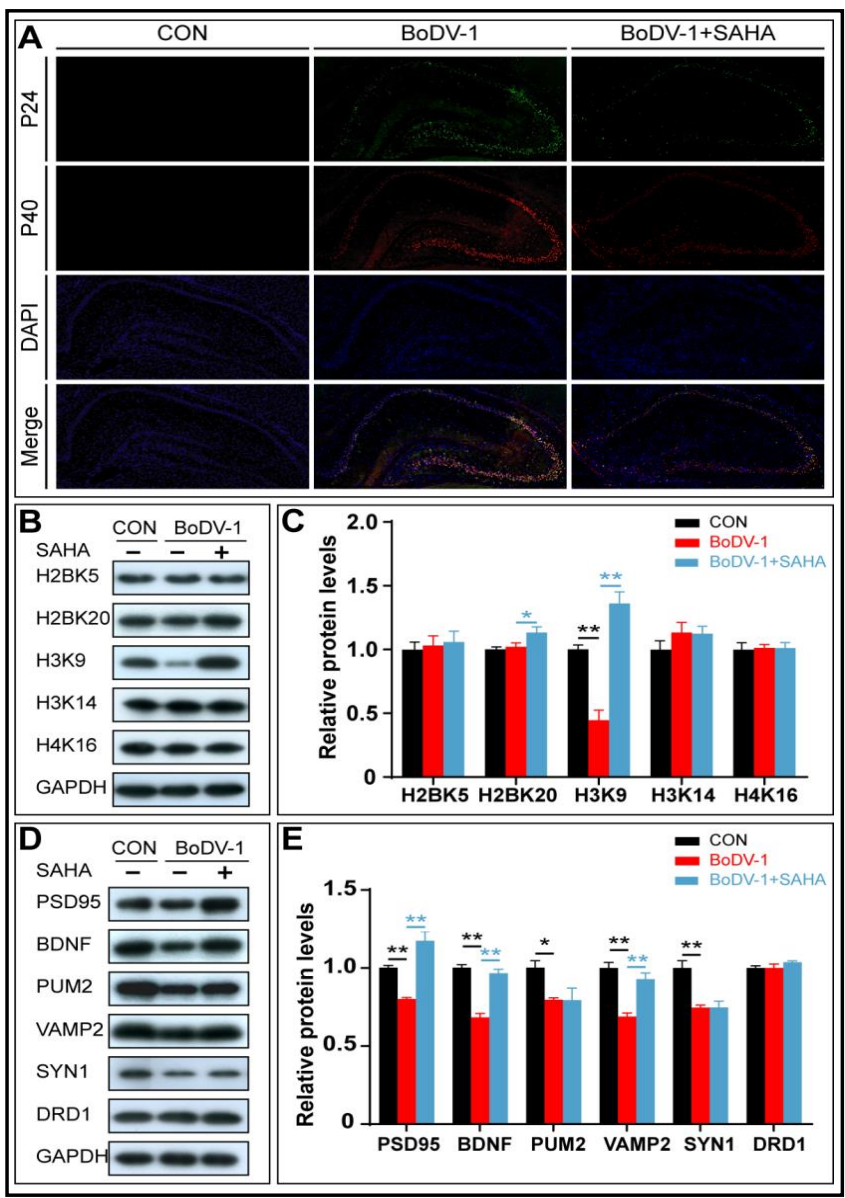

Fig. 5. BoDV-1 infection decreases H3K9ac and affects synaptic protein expression in rats hippocampus. SAHA treatment restores BoDV-1-induced changes. (A) BoDV-1 infection was measured by IFA. BoDV-1 P24 detected with a primary monoclonal antibody (green), BoDV-1 P40 detected with a primary monoclonal antibody (red), Nuclei stained with DAPI (blue), merged image. (B) Levels of histone acetylation were evaluated by Western blots for H2, H3, and H4. (C) Quantification of histone acetylation levels. (D) Western blot analysis of differential expression of PSD95, BDNF, PUM2, VAMP2, SYN1 and DRD1. (E) Quantification of synaptic protein expression levels. GAPDH was used as a loading control. All data are presented as mean \pm SEM. Statistical analysis was performed by unpaired t-test. ${ }^{*} \mathrm{P}<0.05,{ }^{* *} \mathrm{P}<0.01$ vs. controls. 
Fig. 6. BoDV-1 infection caused abnormalities of synaptic plasticity in rats and SAHA treatment make it back to normal. (A) Representative Golgi-impregnated images of the hippocampal region (scale bar: $500 \mu \mathrm{m})$. (B, C) Sholl analysis and quantification of the number of dendritic branches (scale bar: 50 $\mu \mathrm{m})$. (D, E) Representative Golgi staining image (scale bar: $10 \mu \mathrm{m}$ ) and quantification of the dendritic spine density on tertiary branches. All data are presented as mean \pm SEM. Statistical analysis was performed by unpaired t-test. ${ }^{*} \mathrm{P}<0.05,{ }^{* *} \mathrm{P}<0.01$ vs. controls.
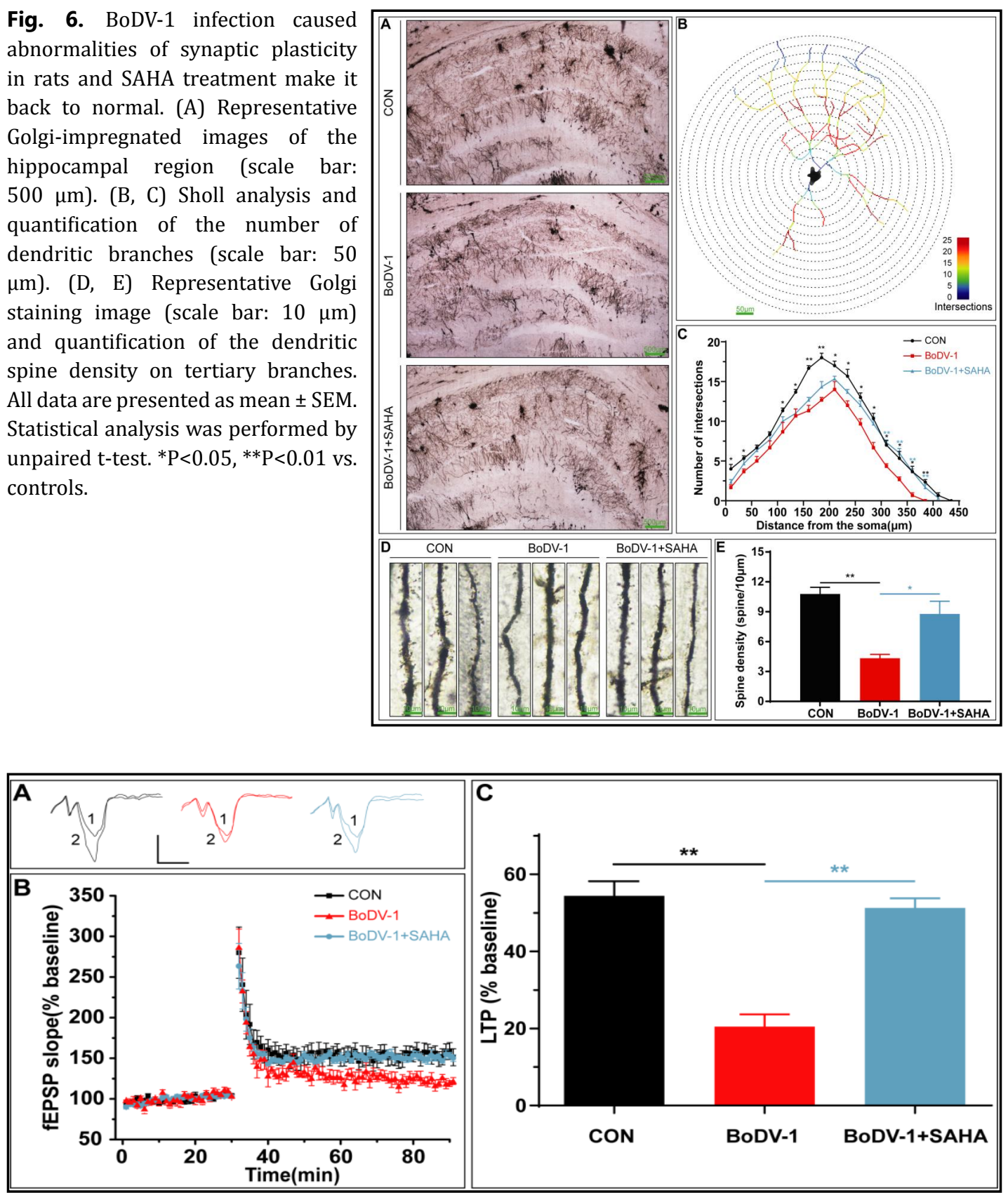

Fig. 7. SAHA rescues the BoDV-1-induced impairment of LTP in CA1of the hippocampus. Representative fEPSP traces (A) and plots of the normalized slopes (B) of the fEPSP 5 min before and 55 min after HFS delivery. (C) The bar graphs of the average percentage changes in the fEPSP slope 50-60 min after HFS delivery. All data are presented as mean \pm SEM. Statistical analysis was performed by One-way ANOVA. ${ }^{* * P}$ $<0.01$ vs. controls ( $n=6-8$ slices from 5 different rats).

\section{BoDV-1 infection impaired hippocampal CA1 LTP and SAHA rescued the impairment in rats}

Hippocampal Long-term potentiation (LTP) is a well-characterized form of synaptic plasticity, and plays a critical role in learning and memory [32]. We detected the influence of BoDV-1 and SAHA on LTP in rats. As shown in Fig. 7, HFS induced a reliable LTP in the control rats (CON: $n=8,159.1 \pm 7.6 \%$ ), whereas the LTP was significantly impaired in the slices from BoDV-1 treated mice (BoDV-1: $\mathrm{n}=6,133.9 \pm 6.4 \%, \mathrm{P}<0.01$ ). As expected, SAHA treatment 


\section{Cellular Physiology Cell Physiol Biochem 2018;49:381-394 \\ \begin{tabular}{ll|l} 
DOI: 10.1159/000492890 & (0) 2018 The Author(s). Published by S. Karger AG, Basel \\
www.karger.com/cpb
\end{tabular} \\ Jie et al.: H3K9 Acetylation in Borna Disease Virus 1-induced Cognitive Impairment}

rescued BoDV-1-induced LTP impairment (SAHA: $n=7,155.2 \pm 7.3 \%, \mathrm{P}<0.01$ ). These results indicate that BoDV-1 treatment impaired hippocampal CA1 LTP, and SAHA significantly rescued the impairment.

\section{Discussion}

Neonatal BoDV-1 infection results in hippocampal alterations and memory dysfunction. Modification of histone acetylation has been implicated in various cognitive processes and multiple neurological disorders [33]. Histone acetylation at lysine residues by HATs causes the remodeling of chromatin structure to an "open" state that promotes gene expression; in contrast, histone deacetylation by HDACs is often associated with transcriptional repression $[34,35]$. Although there have been extensive studies on BoDV-1-driven pathophysiology, the effect of BoDV-1 infection on chromosome plasticity and histone modification remains elusive.

In this study, we found that BoDV-1 infection significantly reduced the level of H3K9 acetylation in primary cultures of hippocampal neurons and in BoDV-1-infected rats. A previous study revealed that environmental enrichment significantly increased the levels of acetyl-H3K9 in the hippocampus and cortex of CK-p25 rats, and caused enhanced learning and memory [36]. In addition, in a model of iron-induced memory impairment, levels of $\mathrm{H} 3 \mathrm{~K} 9$ acetylation were significantly reduced in the hippocampus of rats with neonatal iron overload, but there were no changes in the acetylation levels of H3K14ac, H4K5ac, and H4K12ac [37]. A recent study has also shown that suppressed gene expression and impaired memory are related to the downregulation of histone acetylation of H3K9 and H3K14 [38]. H3K9 is likely to be a target of HDAC1 or 3, which play crucial roles as negative regulators of long-term memory [39]. Our study shows that BoDV-1 infection causes abnormal H3K9 acetylation and memory impairment.

Using ChIP-seq analysis, we found a causative link between H3K9 acetylation deficits and the downregulation of BDNF and PSD95 in BoDV-1-infected neurons. The mRNA and protein levels of BDNF and PSD95 were down-regulated in BoDV-1-infected SD rats. Inhibition of HADC by SAHA treatment rescued the BoDV-1-induced abnormalities in H3K9 acetylation and gene expression. SAHA didn't inhibit the replication of the virus. This also indicates that BoDV-1 causes synaptic plasticity-related damage through abnormal acetylation.

It is well known that BDNF and PSD95 are closely related to memory formation and synaptic plasticity. BDNF is a member of a family of neurotrophic factors critically involved in regulating the survival and differentiation of neurons [40], and in increasing dendritic spine density in an ERK-dependent manner [41]. Moreover, hippocampal-specific deletion of the BDNF gene impairs novel object recognition and spatial learning in the water maze [42]. PSD95 is expressed in the hippocampus and prefrontal cortex and plays a key role in controlling both synaptic strength $[43,44]$ and synaptic plasticity at excitatory synapses [45]. Decreased levels of PSD95 can lead to the disruption of synaptic structures [46] and neuronal apoptosis [47]. BDNF and PSD95 are regulated by H3K9ac. In rat hippocampal cultures, the HDAC-inhibitor, TSA, increases H3K9 histone acetylation, promotes BDNF exon I transcription, and rescues age-dependent decline in hippocampal LTP and BDNF gene expression [48].

Finally, Morris water maze testing showed that BoDV-1 caused spatial memory impairment. We discovered that the mean dendritic spine density and the number of dendritic branches were sharply decreased in the hippocampus of BoDV-1 group rats. In addition, electrophysiological tests showed that BoDV-1 infection reduced the induction of LTP in the hippocampus and impaired synaptic plasticity. However, treatment with SAHA attenuated these negative effects of BoDV-1. These findings further demonstrate that the regulation of H3K9 histone acetylation may play important roles in BoDV-1-induced spatial memory impairment, whereas an HDAC inhibitor may confer protection against BoDV-1induced impairments in spatial memory and hippocampal functions (Fig. 8). An abnormal 


\section{Cellular Physiology Cell Physiol Biochem 2018;49:381-394

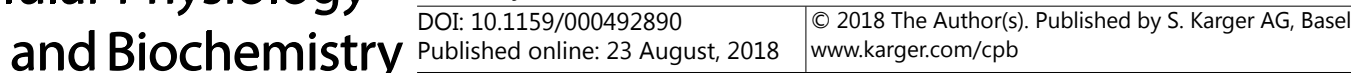

BoDV-1-induced H3K9 acetylation level may impair synaptic plasticity through PSD95 and BDNF, thereby damaging memory formation.

In conclusion, abnormal BoDV-1induced H3K9 acetylation levels may impair synaptic plasticity through PSD95 and BDNF, thereby damaging memory formation. Our study reveals a novel mechanism by which BoDV-1 infection causes cognitive impairments from the perspective of histone modification.

\section{Acknowledgements}

This research was financially supported by The National Key Research and Development Program of China (Grant No. 2017YFA0505700), and the Natural Science Foundation of China (Grant No. 81601207). W.S. is the holder of the Tier 1 Canada Research Chair in Alzheimer's Disease.

\section{Disclosure Statement}

The authors declare no conflict of interests.

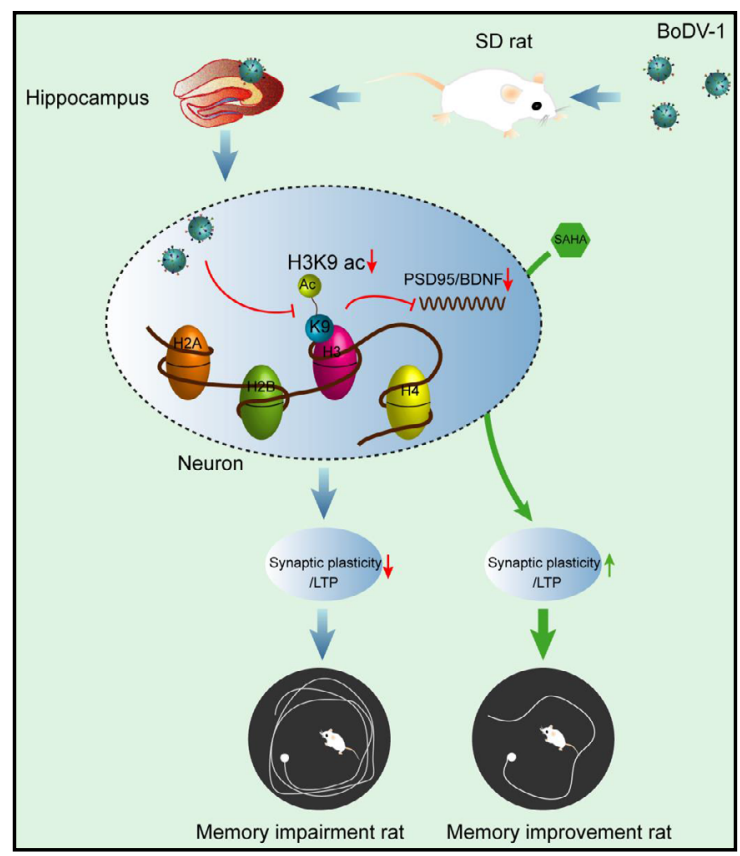

Fig. 8. Schematic diagram illustrates how BoDV-1 induces memory decline in SD rats. We propose that BoDV-1 decreases acetylated histone H3K9 and thus the enrichment in the promoter region of PSD95 and BDNF. This leads to the lower transcription and translation of PSD95 and BDNF in the hippocampus that impair synaptic plasticity, eventually cause memory damage.

\section{References}

$>1$ Lipkin WI, Briese T, Hornig M: Borna disease virus - fact and fantasy. Virus Res 2011;162:162-172.

2 Kuhn JH, Durrwald R, Bao Y, Briese T, Carbone K, Clawson AN, deRisi JL, Garten W, Jahrling PB, Kolodziejek J, Rubbenstroth D, Schwemmle M, Stenglein M, Tomonaga K, Weissenbock H, Nowotny N: Taxonomic reorganization of the family Bornaviridae. Arch Virol 2015;160:621-632.

-3 Amarasinghe GK, Bao Y: Taxonomy of the order Mononegavirales: update 2017. Arch Virol 2017;162:24932504.

4 Pletnikov MV, Moran TH, Carbone KM: Borna disease virus infection of the neonatal rat: developmental brain injury model of autism spectrum disorders. Front Biosci 2002;7:d593-607.

-5 Zhang L, Wang X, Zhan Q, Wang Z, Xu M, Zhu D, He F, Liu X, Huang R, Li D, Lei Y, Xie P: Evidence for natural Borna disease virus infection in healthy domestic animals in three areas of western China. Arch Virol 2014;159:1941-1949.

6 European Centre for Disease Prevention and Control. Acute encephalitis associated with infection with Borna disease virus 1 - Germany, 2018. 26 March 2018. Stockholm: ECDC 2018. URL: http://www.ecdc. europa.eu/sites/portal/files/documents/09-03-2018-RRA-Borna\%20disease\%20virus-Germany.pdf.

$\rightarrow 7$ Liu X, Bode L, Zhang L, Wang X, Liu S, Zhang L, Huang R, Wang M, Yang L, Chen S, Li Q, Zhu D, Ludwig H, Xie $\mathrm{P}$ : Health care professionals at risk of infection with Borna disease virus - evidence from a large hospital in China. Virol J 2015;12:39.

8 Tizard I, Shivaprasad HL, Guo J, Hameed S, Ball J, Payne S: The pathogenesis of proventricular dilatation disease. Anim Health Res Rev 2016;17:110-126. 


\section{Cellular Physiology Cell Physiol Biochem 2018;49:381-394 \begin{tabular}{l|l|l} 
and Biochemistry Published online: 23 August, 2018 & $\begin{array}{l}\text { @ } 2018 \text { The Author(s). Published by S. Karger AG, Basel } \\
\text { www.karger.com/cpb }\end{array}$ \\
\hline
\end{tabular}}

99 Mao Q Zhang L, Guo Y, Sun L, Liu S, He P, Huang R, Sun L, Chen S, Zhang H, Xie P: Identification of suitable reference genes for BDV-infected primary rat hippocampal neurons. Mol Med Rep 2016;14:5587-5594.

10 Borrelli E, Nestler EJ, Allis CD, Sassone-Corsi P: Decoding the epigenetic language of neuronal plasticity. Neuron 2008;60:961-974.

11 Levenson JM, Sweatt JD: Epigenetic mechanisms in memory formation. Nat Rev Neurosci 2005;6:108-118.

12 Sweatt JD: Experience-dependent epigenetic modifications in the central nervous system. Biol Psychiatry 2009;65:191-197.

$>13$ Jarome TJ, Lubin FD: Epigenetic mechanisms of memory formation and reconsolidation. Neurobiol Learn Mem 2014;115:116-127.

14 Yang XJ, Seto E: HATs and HDACs: from structure, function and regulation to novel strategies for therapy and prevention. Oncogene 2007;26:5310-5318.

15 Schneider A, Chatterjee S, Bousiges O, Selvi BR, Swaminathan A, Cassel R, Blanc F, Kundu TK, Boutillier AL: Acetyltransferases (HATs) as targets for neurological therapeutics. Neurotherapeutics 2013;10:568-588.

16 Wu Q, Xu W, Cao L, Li X, He T, Wu Z, Li W: SAHA treatment reveals the link between histone lysine acetylation and proteome in nonsmall cell lung cancer A549 Cells. J Proteome Res 2013;12:4064-4073.

17 Levenson JM, O’Riordan KJ, Brown KD, Trinh MA, Molfese DL, Sweatt JD: Regulation of histone acetylation during memory formation in the hippocampus. J Biol Chem 2004;279:40545-40559.

18 Alarcon JM, Malleret G, Touzani K, Vronskaya S, Ishii S, Kandel ER, Barco A: Chromatin acetylation, memory, and LTP are impaired in CBP+/- mice: a model for the cognitive deficit in Rubinstein-Taybi syndrome and its amelioration. Neuron 2004;42:947-959.

19 Kilgore M, Miller CA, Fass DM, Hennig KM, Haggarty SJ, Sweatt JD, Rumbaugh G: Inhibitors of class 1 histone deacetylases reverse contextual memory deficits in a mouse model of Alzheimer's disease. Neuropsychopharmacology 2010;35:870-880.

-20 Suberbielle E, Stella A, Pont F, Monnet C, Mouton E, Lamouroux L, Monsarrat B, Gonzalez-Dunia D: Proteomic analysis reveals selective impediment of neuronal remodeling upon Borna disease virus infection. J Virol 2008;82:12265-12279.

-21 Bonnaud EM, Szelechowski M, Betourne A, Foret C, Thouard A, Gonzalez-Dunia D, Malnou CE: Borna disease virus phosphoprotein modulates epigenetic signaling in neurons to control viral replication. J Virol 2015;89:5996-6008.

22 Liu X, Zhao L, Yang Y, Bode L, Huang H, Liu C, Huang R, Zhang L, Wang X, Zhang L, Liu S, Zhou J, Li X, He T, Cheng Z, Xie P: Human borna disease virus infection impacts host proteome and histone lysine acetylation in human oligodendroglia cells. Virology 2014;464-465:196-205.

23 Zhao M, Sun L, Chen S, Li D, Zhang L, He P, Liu X, Zhang L, Zhang H, Yang D, Huang R, Xie P: Borna disease virus infection impacts microRNAs associated with nervous system development, cell differentiation, proliferation and apoptosis in the hippocampi of neonatal rats. Mol Med Rep 2015;12:3697-3703.

24 Bode L, Durrwald R, Rantam FA, Ferszt R, Ludwig H: First isolates of infectious human Borna disease virus from patients with mood disorders. Mol Psychiatry 1996;1:200-212.

25 Benito E, Urbanke H, Ramachandran B, Barth J, Halder R, Awasthi A, Jain G, Capece V, Burkhardt S, Navarro-Sala M, Nagarajan S, Schutz AL, Johnsen SA, Bonn S, Luhrmann R, Dean C, Fischer A: HDAC inhibitor-dependent transcriptome and memory reinstatement in cognitive decline models. J Clin Invest 2015;125:3572-3584.

-26 Tokunaga T, Yamamoto Y, Sakai M, Tomonaga K, Honda T: Antiviral activity of favipiravir (T-705) against mammalian and avian bornaviruses. Antiviral Res 2017;143:237-245.

-27 Koshibu K, Graff J, Beullens M, Heitz FD, Berchtold D, Russig H, Farinelli M, Bollen M, Mansuy IM: Protein phosphatase 1 regulates the histone code for long-term memory. J Neurosci 2009;29:13079-13089.

28 Chen L, Huang Z, Du Y, Fu M, Han H, Wang Y, Dong Z: Capsaicin Attenuates Amyloid-beta-Induced Synapse Loss and Cognitive Impairments in Mice. J Alzheimers Dis 2017;59:683-694.

29 Dittrich W, Bode L, Ludwig H, Kao M, Schneider K: Learning deficiencies in Borna disease virus-infected but clinically healthy rats. Biol Psychiatry 1989;26:818-828.

30 Sauder C, Wolfer DP, Lipp HP, Staeheli P, Hausmann J: Learning deficits in mice with persistent Borna disease virus infection of the CNS associated with elevated chemokine expression. Behav Brain Res 2001;120:189-201.

-31 Lee BM, Mahadevan LC: Stability of histone modifications across mammalian genomes: implications for 'epigenetic' marking. J Cell Biochem 2009;108:22-34. 


\section{Cellular Physiology Cell Physiol Biochem 2018;49:381-394

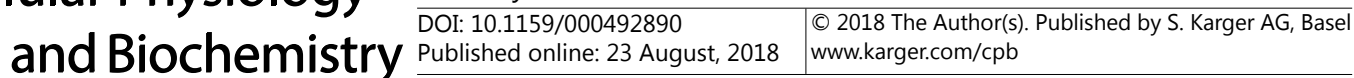

-32 Pastalkova E, Serrano P, Pinkhasova D, Wallace E, Fenton AA, Sacktor TC: Storage of spatial information by the maintenance mechanism of LTP. Science 2006;313:1141-1144.

-33 Jarome TJ, Thomas JS, Lubin FD: The epigenetic basis of memory formation and storage. Prog Mol Biol Transl Sci 2014;128:1-27.

-34 Saha RN, Pahan K: HATs and HDACs in neurodegeneration: a tale of disconcerted acetylation homeostasis. Cell Death Differ 2006;13:539-550.

-35 Peserico A, Simone C: Physical and functional HAT/HDAC interplay regulates protein acetylation balance. J Biomed Biotechnol 2011;2011:371832.

-36 Fischer A, Sananbenesi F, Wang X, Dobbin M, Tsai LH: Recovery of learning and memory is associated with chromatin remodelling. Nature 2007;447:178-182.

-37 Silva PF, Garcia VA, Dornelles Ada S, Silva VK, Maurmann N, Portal BC, Ferreira RD, Piazza FC, Roesler $\mathrm{R}$, Schroder N: Memory impairment induced by brain iron overload is accompanied by reduced H3K9 acetylation and ameliorated by sodium butyrate. Neuroscience 2012;200:42-49.

38 Singh P, Konar A, Kumar A, Srivas S, Thakur MK: Hippocampal chromatin-modifying enzymes are pivotal for scopolamine-induced synaptic plasticity gene expression changes and memory impairment. J Neurochem 2015;134:642-651.

-39 McQuown SC, Barrett RM, Matheos DP, Post RJ, Rogge GA, Alenghat T, Mullican SE, Jones S, Rusche JR, Lazar MA, Wood MA: HDAC3 is a critical negative regulator of long-term memory formation. J Neurosci 2011;31:764-774.

40 Huang EJ, Reichardt LF: Neurotrophins: roles in neuronal development and function. Annu Rev Neurosci 2001;24:677-736.

41 Alonso M, Medina JH, Pozzo-Miller L: ERK1/2 activation is necessary for BDNF to increase dendritic spine density in hippocampal CA1 pyramidal neurons. Learn Mem 2004;11:172-178.

-42 Heldt SA, Stanek L, Chhatwal JP, Ressler KJ: Hippocampus-specific deletion of BDNF in adult mice impairs spatial memory and extinction of aversive memories. Mol Psychiatry 2007;12:656-670.

43 Elias GM, Funke L, Stein V, Grant SG, Bredt DS, Nicoll RA: Synapse-specific and developmentally regulated targeting of AMPA receptors by a family of MAGUK scaffolding proteins. Neuron 2006;52:307-320.

44 Zhang P, Lisman JE: Activity-dependent regulation of synaptic strength by PSD-95 in CA1 neurons. J Neurophysiol 2012;107:1058-1066.

45 Migaud M, Charlesworth P, Dempster M, Webster LC, Watabe AM, Makhinson M, He Y, Ramsay MF, Morris RG, Morrison JH, O'Dell TJ, Grant SG: Enhanced long-term potentiation and impaired learning in mice with mutant postsynaptic density-95 protein. Nature 1998;396:433-439.

46 Rubino T, Realini N, Braida D, Guidi S, Capurro V, Vigano D, Guidali C, Pinter M, Sala M, Bartesaghi R, Parolaro D: Changes in hippocampal morphology and neuroplasticity induced by adolescent THC treatment are associated with cognitive impairment in adulthood. Hippocampus 2009;19:763-772.

-47 Sultana R, Banks WA, Butterfield DA: Decreased levels of PSD95 and two associated proteins and increased levels of $\mathrm{BCl} 2$ and caspase 3 in hippocampus from subjects with amnestic mild cognitive impairment: Insights into their potential roles for loss of synapses and memory, accumulation of Abeta, and neurodegeneration in a prodromal stage of Alzheimer's disease. J Neurosci Res 2010;88:469-477.

-48 Tian F, Marini AM, Lipsky RH: Effects of histone deacetylase inhibitor Trichostatin A on epigenetic changes and transcriptional activation of Bdnf promoter 1 by rat hippocampal neurons. Ann N Y Acad Sci 2010;1199:186-193. 